\title{
Distribution of Return Intervals of Extreme Events
}

\author{
Cecilia Pennetta \\ Dipartimento di Ingegneria dell'Innovazione, Università di Lecce and CNR-INFM, \\ National Nanotechnology Laboratory, Via Arnesano, I-73100, Lecce, Italy, \\ cecilia.pennetta@unile.it
}

\begin{abstract}
The distribution of return intervals of extreme events is studied in time series characterized by finite-term correlations with non-exponential decay. Precisely, it has been analyzed the statistics of the return intervals of extreme values of the resistance fluctuations displayed by resistors with granular structure in nonequilibrium stationary states. The resistance fluctuations are calculated by Monte Carlo simulations using a resistor network approach. It has been found that for highly disordered networks, when the auto-correlation function displays a non-exponential and non-power-law decay, the distribution of return intervals of the extreme values is a stretched exponential, with exponent independent of the threshold.
\end{abstract}

PACS numbers: 05.40.-a, 05.45.Tp, 02.50.-r 


\section{INTRODUCTION}

Fluctuations of prices in financial markets, wind speed data or daily precipitations in a given place for the same time windows are typically described by time series $x(t)$ made by uncorrelated records ${ }^{1,2}$. In these cases, by considering the return intervals $r_{q}$ of extreme events associated with the overcoming of a given threshold $q$ (i.e. the time intervals between two consecutive occurences of the condition $x(t)>q$ ), it can be seen that the $r_{q}$ are exponentially distributed ${ }^{1,3,4}$. In other terms, the probability density function (PDF) of the distribution of the $r_{q}$ is given by ${ }^{1,3,4}$ :

$$
P_{q}(r)=\left(1 / R_{q}\right) \exp \left(-r / R_{q}\right)
$$

where $R_{q}$ is the mean return interval. Of course, the higher the threshold $q$, the bigger is the value of $R_{q}$. On the other hand, in the last years it has become clear that several other important examples of time series display long-term correlations ${ }^{2,5,6}$. This is the case of physiological data (heartbeats ${ }^{7,8}$ and neuron spikes ${ }^{9}$ ), hydro-meteorological records (daily temperatures ${ }^{2,5,10}$ ), geophysical or astrophysical data (occurrence of earthquakes ${ }^{11,12}$ or solar flares ${ }^{13}$ ), internet traffic ${ }^{5}$ and stock market volatility ${ }^{2,14}$ records. Long-term correlated series are characterized by an auto-correlation function, $C_{x}(s)$, decaying as a power-law:

$$
C_{x}(s)=<x_{i} x_{i+s}>=\frac{1}{N-s} \sum_{i=1}^{N-s} x_{i} x_{i+s} \sim s^{-\gamma_{x}}
$$

with exponent $\gamma_{x}$ (correlation exponent) between 0 and 1 . In this case, the mean correlation time $\tau$, given by the integral over $s$ of $C_{x}(s)$, diverges. The effect of long-terms correlations on the statistics of the $r_{q}$ has been first studied by Bunde et al. ${ }^{5}$ and the conclusions of this study were the following. i) The mean return interval $R_{q}$ is left unchanged by the presence of longterms correlations. ii) The distribution of return intervals becomes a stretched exponential:

$$
P_{q}(r)=a \exp \left[-\left(b r / R_{q}\right)^{\gamma}\right]
$$

where the two exponents $\gamma$ and $\gamma_{x}$ were found to be equal. iii) The return intervals themself are long-term correlated with a correlation exponent $\gamma^{\prime} \approx \gamma_{x}$. As noted by Altmann 
and Kantz in a very recent paper ${ }^{2}$, the result i), obtained by Bunde et al. ${ }^{5}$ by statistical arguments, can be identified with Kac's lemma ${ }^{15}$ introduced in the context of dynamical systems. The results ii) and iii) were obtained in Ref. ${ }^{5}$ on the ground of numerical calculations performed on long-term correlated and Gaussian time series generated by the algorithm described in Ref. ${ }^{16}$. It must be noted that the result ii) only applies to linear time series (i.e. to series whose properties are completely defined by the power spectrum and by the probability distribution, regardless of the Fourier phases) $)^{2,6}$. Apart from this restriction, the stretched exponential distribution of the $r_{q}$ seems to be a general feature in presence of long-term correlations in a time series ${ }^{2,6}$. It must be underlined that this fact has important consequences on the observation of extreme events: indeed it implies a strong enhancement of the probability of having return intervals well below and well above $R_{q}$, in comparison with the occurrence of extreme events in an uncorrelated time series. Furthermore, it must be noted that the stretched exponential distribution depends on the parameter $\gamma$ only, being $a$ and $b$ in Eq. (3) functions of $\gamma$, as shown in Ref. ${ }^{2}$.

In this paper it will be analyzed the effect on the distribution of the $r_{q}$ of the presence of finite-term correlations with non-exponential decay, a situation which can occur in systems which are approaching criticality, where intermediate behaviors, consisting in a non-exponential and non-power-law decay of correlations can emerge ${ }^{4}$. Precisely, in the following section it will be studied the statistics of the return intervals of extreme values of the resistance fluctuations displayed by a resistor with granular structure in a nonequilibrium stationary state ${ }^{17-21}$.

\section{METHOD AND RESULTS}

The time series analyzed in this paper consist in the resistance fluctuations of a thin resistor with granular structure, in contact with a thermal bath at temperature $T_{0}$ and biased by an external current $I$. The resistance values are calculated by using the stationary and biased resistor network (SBRN) model ${ }^{17-22}$. This model describes a thin film with granular 
structure as a two-dimensional resistor network in a stationary state determined by the competition between two stochastic processes, breaking and recovery of the elementary resistors. A broken elementary resistor (with resistance $10^{9}$ higher than the resistance corresponding to a normal elementary resistor) can be associated with a high resistivity region within the conducting material. Both processes are thermally activated and biased by the external current. The resistance of the network and its fluctuations are calculated by Monte Carlo simulations ${ }^{17,18,21}$. Within this model, the level of intrinsic disorder in the network (average fraction of broken resistors in the vanishing current limit ${ }^{23}$ ) is controlled by a characteristic parameter $^{20}: \lambda \equiv\left(E_{D}-E_{R}\right) / k_{B} T_{0}$, where $E_{D}$ and $E_{R}$ are the activation energies respectively of the breaking and recovery processes. The intrinsic disorder parameter $\lambda$ ranges between: $\lambda_{\min }<\lambda<\lambda_{\max }$, where $\lambda_{\max }$ corresponds to an homogeneous resistor (perfect network) and $\lambda=\lambda_{\min } \approx 0$ to the maximum level of intrinsic disorder compatible with a stationary state of the network (stationary resistance fluctuations) ${ }^{18^{-21,23}}$.

In addition to this intrinsic disorder, the SBRN model considers also the presence of a disorder driven by the external current $I$. As a consequence, for a given value of $\lambda$, and for a network of given size, nonequilibrium stationary states of the network exist only when $I \leq I_{B}$ (breakdown threshold). For contrast, when $I>I_{B}$ the network undergoes an electrical breakdown associated with an irreversible divergence of its resistance $\mathrm{e}^{1-19,21}$. For an arbitrary value of $\lambda$ this breakdown corresponds to a first order transition from a conducting to a non-conducting state of the network ${ }^{18^{-20}}$. However, at decreasing $\lambda$ values, when $\lambda \rightarrow \lambda_{\text {min }}$, the system becomes more and more close to its critical point ${ }^{18^{-20}}$.

All the details about the SBRN model and its results can be found in Refs. ${ }^{17-21}$. However, it must be noted that this model provides a good description of many features associated with nonequilibrium stationary states and with the electrical instability of composites and granular materials ${ }^{17-19,24}$, including the electromigration damage of metallic lines ${ }^{21}$. Finally, it must be underlined that, apart from the specific system described by the SBRN model, the method adopted here for generating the time series can be also viewed as a pure numerical algorithm for generating numerical series with different and tunable correlation properties. 


\section{FIGURES}

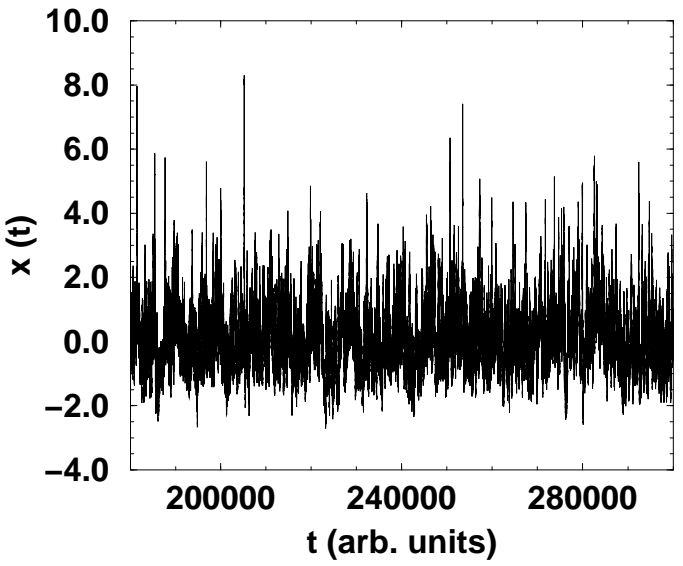

FIG. 1. Normalized resistance fluctuations versus time (this last is expressed in simulation steps). The resistance values have been normalized to provide a zero average and a unit variance for $x(t)$.

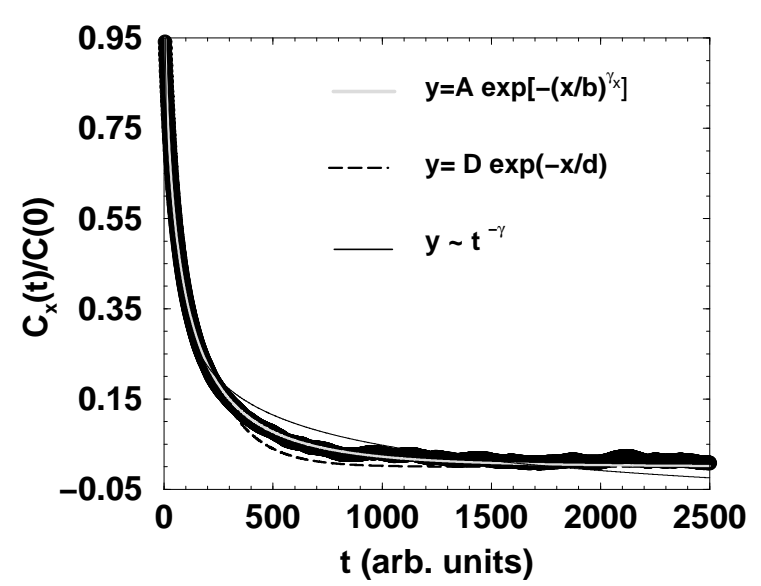

FIG. 2. Auto-correlation function of the time series in Fig. 1 (black-thick curve). The grey, black-dashed and black-solid curves represent the best-fit with a stretched exponential, an exponential and a power-law, respectively.

Then, by indicating with $R$ the resistance of the network (expressed in $\Omega$ ) and with $t$ the time (expressed in iteration steps), long $R(t)$ time series (typically made of $1 \div 2 \times 10^{6}$ records) have been generated and analyzed for different values of $\lambda$, of the external current and of the network size. Precisely, normalized series with zero average and unit variance have been considered: $x(t) \equiv(R(t)-<R>) / \sigma$, where $<R>$ is the average value of the network resistance and $\sigma$ the root-mean-square deviation from the average. The analysis has been performed by calculating the auto-correlation function and the PDF of the $x$ records, the return intervals $r_{q}$ of the extreme values for different threshold $q$ and their distribution $P_{q}(r)$ (the values of $q$ are expressed in units of $\sigma$ ). For small $\lambda$ values (high level of intrinsic disorder), it has been found that $C_{x}$ displays a non-exponential and non-power-law decay. This behavior is different from that obtained for high $\lambda$ values (low level of intrinsic disorder), where $C_{x}$ decays exponentially (consistently with the Lorentzian power spectrum reported in Refs. $\left.{ }^{17,18,20}\right)$. By focusing on this situation, of interest for systems which are approaching 
criticality, in the following of this section results will be shown concerning a network of size $125 \times 125$, biased by a current $I=I_{B}=0.011$ A and obtained by taking $\lambda=0.33$.

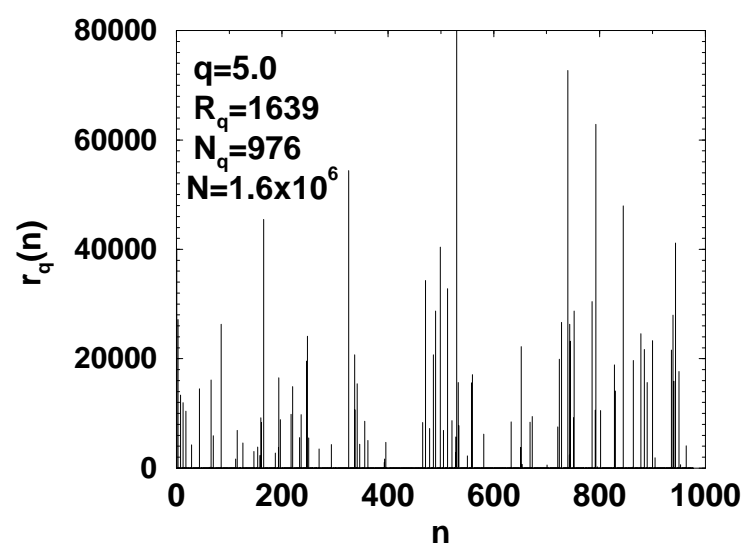

FIG. 3. Return intervals of extreme values above the threshold $q=5$ (in units of root-mean square deviation) for the time series of Fig. 1.

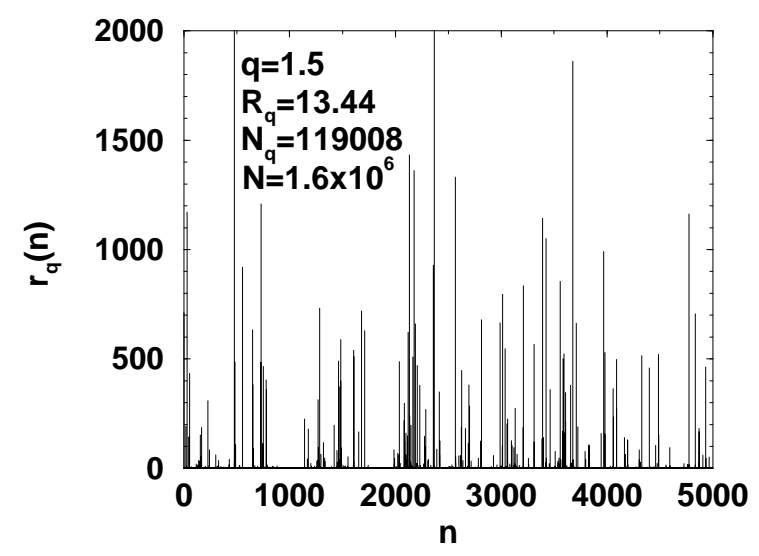

FIG. 4. Return intervals of extreme values above the threshold $q=1.5$ for the same time series of the previous figures. Here only the first 5000 intervals are shown.

The $x(t)$ time series is shown in Fig. 1 (only a small portion of the total number of records, $N=1.6 \times 10^{6}$, is reported in this figure). As evident from Fig. 1, the resistance fluctuations exhibit a strong non-Gaussianity and actually their PDF is well described by the Bramwell-Holdworth-Pinton distribution ${ }^{25}$, as discussed in Refs. ${ }^{19,20}$. The auto-correlation function of $x(t)$ is reported in Fig. 2. The function significantly deviates from a single exponential and from a power-law while it is well fitted by a stretched exponential:

$$
\left.C_{x}(s)=A \quad \exp \left[(-s / b)^{\gamma}\right)\right]
$$

with the following values of the fitting parameters: $A=1.23, b=74.9$ and $\gamma=0.54$. Many other functions have also been considered for the best-fit of the $C_{x}$ data. However, it has been found that the stretched exponential optimizes the best-fit procedure with the minimum numbers of fitting parameters. It must be remarked that a stretched exponential describes a behavior intermediate between a simple exponential decay (which is obtained for $\gamma=1$ ) and a constant behavior (a limit of power-law) for $\gamma \rightarrow 0$. Moreover, the correlation 
time corresponding to the expression (4) of $C_{x}$ is finite.

The sequence of the $N_{q}$ return intervals of the values above the threshold $q=5$ is plotted in Fig. 3 as a sequence of $N_{q}$ impulses. The figure shows that a succession of very short return intervals $\left(r_{q} \ll 100\right.$, the apparently empty portions of the horizontal axis) is followed by a succession of long intervals, indicating a strong clustering of the extreme events, a feature similar to that exhibited by the data of Bunde et al. ${ }^{6}$ which instead concern longterm correlated records and very different from what observed for uncorrelated time $\operatorname{series}^{26}$. Thus, the clustering of the extreme events is present even if the $x$ records are not long-term correlated while they are characterized by a finite correlation time. This clustering of the extreme values persists also by lowering the threshold. This is shown in Fig. 4 which reports the sequence of the return intervals obtained for $q=1.5$.

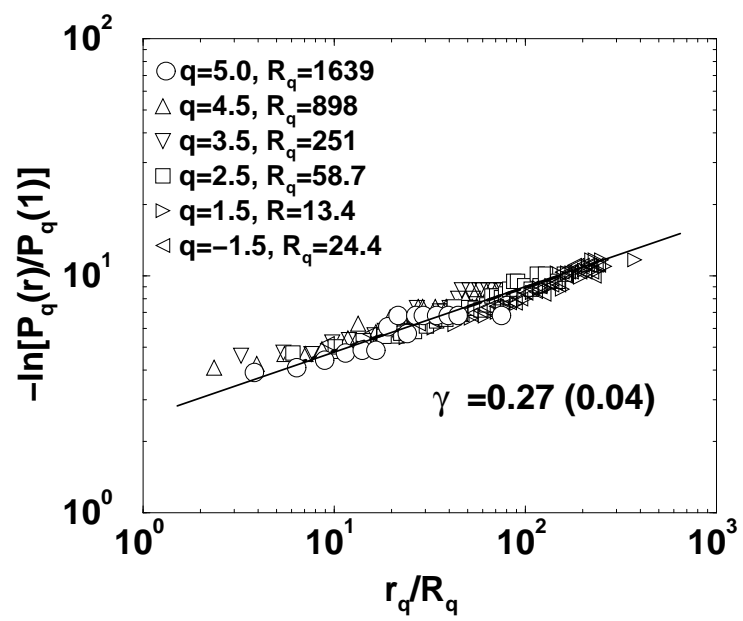

FIG. 5. Double-logarithmic plot of the normalized probability density of the return intervals for different thresholds $q$.

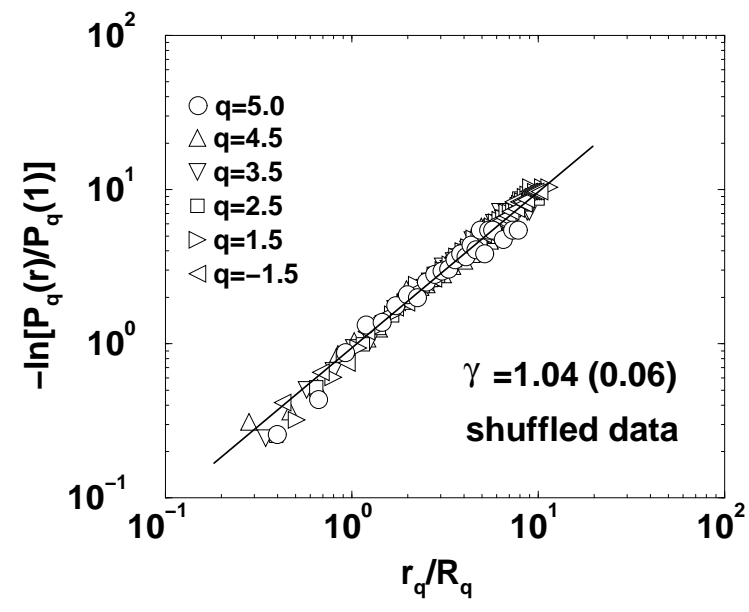

FIG. 6. The same as Fig. 5 but calculated after random shuffling the x-records.

Figure 5 displays the probability density function $P_{q}(r)$ of the distribution of the return intervals as a function of $r_{q} / R_{q}$ for different thresholds $q$ ranging from -1.5 to 5.0 (the probability density has been normalized to $\left.P_{q}(1)\right)$. A double-logarithmic plot of $P_{q}$ has been adopted because in this representation a stretched exponential function with exponent $\gamma$ appears as a straight line of slope $\gamma$. Therefore Fig. 5 shows that the distribution of the 
return intervals of extreme values of the x-series is well described by a stretched exponential and that the value of the exponent $\gamma$ is independent of the threshold $q$ in a large range of $q$-values. This occurs even in absence of long-term corrrelations and in presence of a finite correlation time. This not-obvious result agrees with the conclusions of Altmann and Kantz formulated in their recent paper ${ }^{2}$. For comparison, Fig. 6 reports the probability density function $P_{q}(r)$ of the distribution of the return intervals obtained after random shuffling the records of the same $\mathrm{x}$-series: in this case $\gamma=1$, i.e. the distribution of the $r_{q}$ is exponential, as it must be for uncorrelated time series.

\section{CONCLUSIONS}

The distribution of return intervals of extreme events has been studied in time series with finite-term correlations. Precisely, it has been analyzed the distribution of return intervals of extreme values of the resistance fluctuations displayed by a resistor with granular structure in nonequilibrium stationary states. The resistance fluctuations were calculated by using the SBRN model based on a resistor network approach ${ }^{17-21}$. It has been found that for highly disordered networks, when the auto-correlation function displays a nonexponential and a non-power-law decay, the distribution of the $r_{q}$ is well described by a stretched exponential with exponent $\gamma$ largely independent of the threshold $q$. This result shows that the stretched exponential distribution describes the distribution of the return intervals of extreme events not only when long-term correlations are present in the time series $^{2,5,6}$, but also when finite-term correlations exist among the records, characterized by a non-exponential decay, a situation typical of systems which are approaching criticality.

Partial support from SPOT NOSED project IST-2001-38899 of E.C. and from MIUR cofin-03 project "Modelli e misure in nanostrutture" is acknowledged. The author thanks

S. Ruffo (University of Florence, Italy), P. Olla (ISAC-CNR, Lecce, Italy), G. Salvadori and E. Alfinito (University of Lecce, Italy) for helpful discussions. 


\section{REFERENCES}

${ }^{1}$ H. von Storch and F. W. Zwiers, Statistical Analysis in Climate Research, Cambridge University Press, Cambridge (2001).

${ }^{2}$ E. G. Altmann and H. Kantz, Phys. Rev. E, 71, 056106 (2005).

${ }^{3}$ S. Kotz and S. Nadarajah, Extreme Value Distributions, Theory and Applications, Imperial College Press, London (2002).

${ }^{4}$ D. Sornette, Critical Phenomena in Natural Sciences, Chaos, Fractals, Selforganization and Disorder: Concepts and Tools, Springer, Berlin, (2004).

${ }^{5}$ A. Bunde, J. F. Eichner, S. Havlin and J. W. Kantelhardt, Physica A, 330, 1 (2003).

${ }^{6}$ A. Bunde, J. F. Eichner, J. W. Kantelhardt and S. Havlin, Phys. Rev. Lett., 94, 048701 (2005).

${ }^{7}$ A. Bunde, S. Havlin, J. W. Kantelhardt, T. Penzel, J. H. Peter and K. Voigt, Phys. Rev. Lett., 85, 3736 (2000).

${ }^{8}$ Y. Ashkenazy, P. C. Ivanov, S. Havlin, C. K. Peng, A. L. Goldberger and H. E. Stanley, Phys. Rev. Lett., 86, 1900 (2001).

${ }^{9}$ J. Davidsen and H. G. Schuster, Phys. Rev. E, 65, 026120 (2002).

${ }^{10}$ E. Koscielny-Bunde, A. Bunde, S. Havlin, H. E. Roman, Y. Goldreich and H. J. Schellnhuber, Phys. Rev. Lett., 81, 729 (1998).

${ }^{11}$ P. Bak, K. Christensen, L. Danon and T. Scanlon, Phys. Rev. Lett., 88, 178501 (2002).

12 A. Corral, Phys. Rev. Lett., 92, 108501 (2004).

${ }^{13}$ G. Boffetta, V. Carbone, P. Giuliani, P. Veltri and A. Vulpiani, Phys. Rev. Lett., 83, 4662 (1999).

${ }^{14}$ Y. Liu, P. Cizeau, M. Meyer, C. K. Peng, H. E. Stanley, Physica A, 245, 437, (1997). 
${ }^{15}$ M. Kac, Bull. of the Am. Math. Soc., 53, 1002 (1947).

${ }^{16}$ H. A. Makse, S. Havlin, M. Schwartz and H. E. Stanley, Phys. Rev. E, 53, 5445 (1996).

${ }^{17}$ C. Pennetta, L. Reggiani, G. Trefán and E. Alfinito, Phys. Rev. E, 65, 066119 (2002).

${ }^{18}$ C. Pennetta, Fluct. and Noise Let., 2, R29 (2000).

${ }^{19}$ C. Pennetta, E. Alfinito, L. Reggiani and S. Ruffo, Physica A, 340, 380, (2004).

${ }^{20}$ C. Pennetta, E. Alfinito, L. Reggiani and S. Ruffo, in Noise in Complex Systems and Stochastic Dynamics II, p.38, ed. by Z. Gingl and J. M. Sancho and L. Schimansky-Geier and J. Kertesz, Proceedings of SPIE, 5471, Int. Soc. Opt. Eng., Bellingham (2004).

${ }^{21}$ C. Pennetta, E. Alfinito, L. Reggiani, F. Fantini, I. De Munari and A. Scorzoni, Phys. Rev. B, 70, 174305 (2004).

${ }^{22}$ C. Pennetta, G. Trefán and L. Reggiani, in Unsolved Problems of Noise and Fluctuations, p. 447, ed. by D. Abbott and L. B. Kish, AIP Conf. Procs. 511, New York (1999).

${ }^{23}$ C. Pennetta, L. Reggiani and G. Trefán, Phys. Rev. Lett., 85, 5238 (2000).

${ }^{24}$ C. D. Mukherjee, K. K. Bardhan and M. B. Heaney, Phys. Rev. Lett. 83, 1215 (1999) and C. D. Mukherjee, K. K. Bardhan, Phys. Rev. Lett. 91, 025702 (2003).

${ }^{25}$ S.T. Bramwell, P. C. W. Holdsworth and J. F. Pinton, Nature, 396, 552 (1998).

${ }^{26}$ C. Pennetta and E. Alfinito, in Unsolved Problems of Noise and Fluctuations, ed. by L. Reggiani, C. Pennetta, V. Akimov, E. Alfinito and M. Rosini, AIP Conf. Procs., 800, New York, in print. 